\title{
EXTENDED CHAIN CRYSTALS OF LINEAR HIGH POLYMERS
}

\author{
BERNHARD WUNDERLICH \\ Department of Chemistry, Rensselaer Polytechnic Institute, Troy, N.Y., 12181, \\ USA.
}

\begin{abstract}
Studies of extended chain crystals carried out over the last 10 years have been reviewed. Crystallization in an extended chain conformation is possible either by chain folding followed by extension in the solid state, or by crystallization during polymerization. The equilibrium isometric crystal habit is only rarely exhibited. Crystals which derive from folded chain macroconformation by subsequent chain extension have lamellar habit, crystals which crystallize during polymerization often show fibrillar habit because of nucleation limitation. The morphology of extended chain crystals is analogous to folded chain crystals. Their properties have been shown to be close to the extrapolated equilibrium properties.
\end{abstract}

\section{INTRODUCTION}

The lowest free energy macroconformation of all studied linear homopolymers was shown over the years to be helically extended ${ }^{2}$. Globular conformations are stable only in some copolymers, for example many of the proteins; and, of course, all macromolecules take on globular or coiled conformations at sufficiently high temperatures out of entropy considerations. Any macromolecule with a helical low free energy conformation must in turn on crystallization take on an 'extended chain' crystal structure out of packing considerations. Trying to compute an equilibrium habit using the Wulff theorem ${ }^{2}$ yields for such crystals with any reasonable set of surface free energies forms close to isometric crystals. This is in contrast to most crystallization experiments in polymer science.

The two basic crystallization habits of macromolecules are lamellar and fibrillar, both clearly non-equilibrium habits. Figure $l$ shows a typical lamellar growth spiral of polyethylene grown from solution, and Figure 2 illustrates fibrillar polytetrafluoroethylene grown by gas phase polymerization. In Figure $l$ the polyethylene molecules are normal to the lamellar surface, necessitating their folding every time the surface is reached. The typical macroconformation of polymers grown from solution involves folding every 50 to $500 \AA$ rendering the crystal metastable because of a high surface free energy. The fibrillar habit shown in Figure 2 is in accord with a low free energy molecular arrangement parallel to the fibre axis. The side surface free energy is, however, large enough in fibrillar habits to cause these crystals to be metastable also. In addition, many fibrillar crystals of linear high polymers still contain chain folds. This is 


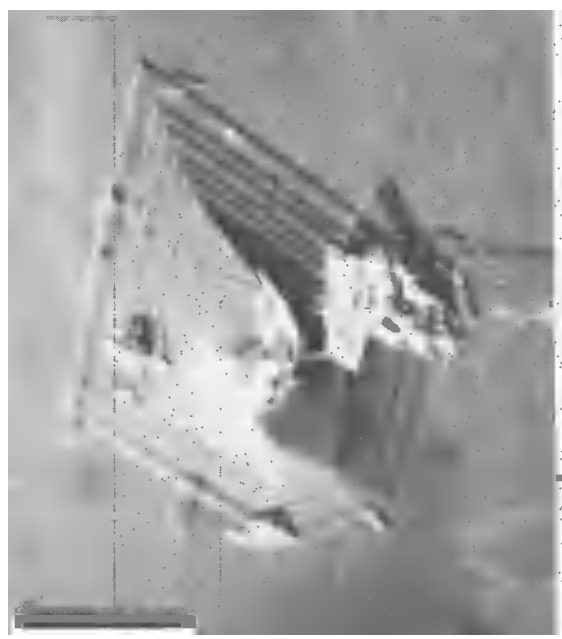

FIG. 1

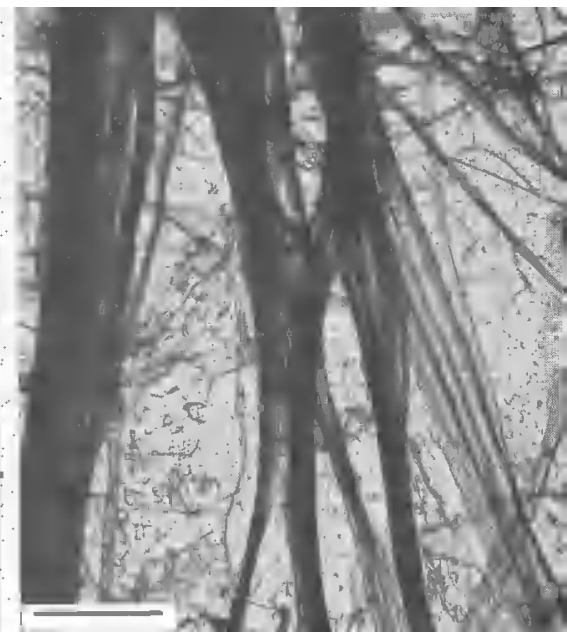

FIG. 2

Figure 1. Interference micrograph of a polyethylene growth spiral from solution. Incident light. Normarski interference optics. Scale $30 \mu \mathrm{m}$. (Sullivan, Thesis. Department of Chemistry, Cornell University. 1963)

Figure 2. Fibrillar polytetrafluoroethylene. Electron micrograph of gas phase polymerized monomer. Transmission electron micrograph. Scale about $5 \mu \mathrm{m}$. (Melillo and Wunderlich. Unpublished. 1967)

particularly true for fibrillar crystals formed during deformation from already polymerized molecules.

This paper deals with the crystallization, morphology, and some properties of extended chain crystals. Since extended helical sections are also present on a small scale in crystals usually termed 'folded', and it is frequently difficult to assess exact degrees of chain extension, we found it necessary to distinguish between folded. extended, and fully extended macroconformation crystals ${ }^{3}$. The dividing line between folded and extended chain crystals is drawn when the separation reaches about $2000 \AA$ between folds. This definition was chosen because such a length corresponds to a molecular weight of at least 20000, a value which is of the order of magnitude of the accepted lower limit for a high polymer molecule. It also represents a length at which effects due to the crystal surface at the chain ends or folds become negligible, independent of reasonable specific surface free energies. For example, an extended-chain single crystal lamella of high molecular weight polyethylene of $2000 \AA$ has a melting point depression of only about $1^{\circ} \mathrm{C}$ due to the fold surface. Finally, for high molecular weight polymers, crystals of about $2000 \AA$ chain extension are difficult to obtain. Crystallization from the melt and usual annealing techniques are, at least for the present, not able to achieve chain extensions above 500 to $1500 \AA$. The methods of producing extended chain crystals to be discussed below are, in contrast, easily able to produce $10000 \AA$ and greater chain extensions. The division at $2000 \AA$ is thus at a level where at present few samples are available making the classification easy. The distinction between extended and fully 
extended chain crystals is obvious. As soon as it has been established that no chain folds are present, this classification can be made. The crystal must in this case have at least the dimension of the helically fully extended molecule in the direction of the helix.

\section{FORMATION OF EXTENDED CHAIN CRYSTALS}

Crystals of macromolecules can be grown by two basic routes. Either an already polymerized flexible macromolecule is crystallized from the molten or dissolved state ${ }^{4}$, or crystallization is carried out during polymerization ${ }^{5}$. Single crystals grown by both routes were investigated quite early in the development of polymer science. Staudinger and Signer $^{6}$ in 1929 grew extended chain single crystals of polyoxymethylene of reasonable molecular weight by crystallization during polymerization from gaseous formaldehyde. In 1938 Storks $^{7}$ grew folded-chain single crystals of gutta percha from dilute chloroform solution. Neither of these observations however received attention for many years. The morphology of crystals grown from the macromolecular melt and solution was only investigated in the early 1960s after growth of folded chain single crystals became well established ${ }^{4,8}$. The major conclusion from the considerable body of information developed on chain folding is that macromolecules once in the random state must fold in order to crystallize. The reason for this initial chain folding rests with the kinetics of the nucleation of each macromolecule on crystallization. Extended chain crystals from the already polymerized molecules can thus only be obtained by secondary chain extension in the solid state after initial chain folding. Crystallization during polymerization from the monomer does not have such an inherent limitation and provides a more direct path to extended chain crystals.

\section{Extended chain crystal growth by chain folding and extension}

The first extended chain crystals grown by initial chain folding followed by extension were reported by Bunn, Cobbold and Palmer in the case of polytetrafluoroethylene ${ }^{9}$. Figure 3 illustrates a fracture surface of an extended chain sample of polytetrafluoroethylene. The large striated lamellae are about 10000 to $50000 \AA$ in thickness with the macromolecular helices arranged parallel to the striations. The lamellar width is several hundred $\mu \mathrm{m}$. Since the molecules are higher in molecular weight in the chosen sample chain folding is still prevalent at the lamellar surface. The crystals were grown by slow cooling from the melt. The process of folding followed by extension can be seen at the lamellar edge illustrated in Figure 4 which shows distinct rounding. new polymer is added to the tip, folded similarly to crystal growth from dilute solution with a fold length of 200 to $500 \AA$. Immediately thereafter, thickening occurs over a crystal width of 2 to $5 \mu \mathrm{m}$ to the final thickness of about $30000 \AA$. The molecules in this particular preparation were about $100000 \AA$ long.

More detailed investigations were carried out on polyethylene ${ }^{10-17}$. An early electronmicrograph of a fracture surface is shown in Figure 5. Chain extension after folding required in this case elevation of pressure. As much as $3000 \mathrm{~atm}$ 


\section{BERNHARD WUNDERLICH}

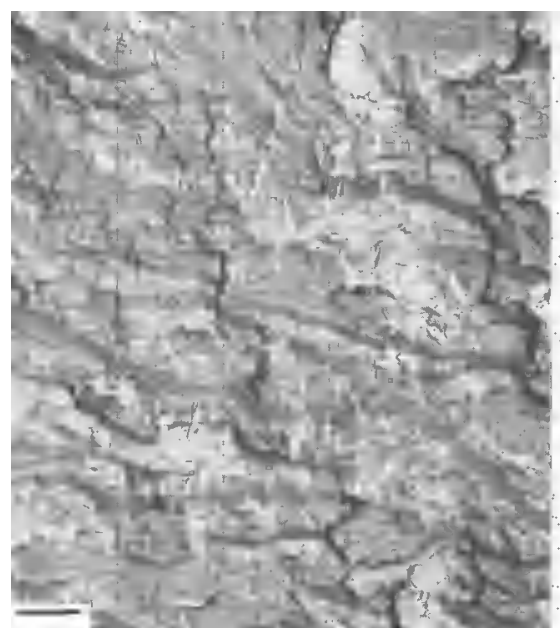

FIG. 3

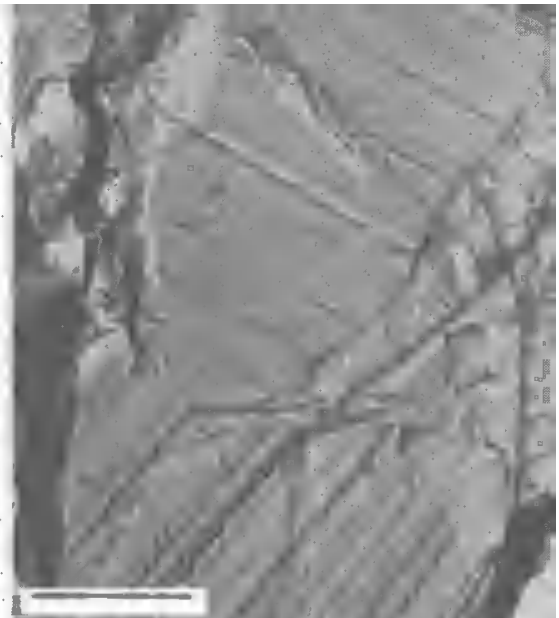

FIG. 4

Figure 3. Electron micrograph of a fracture surface of polytetrafluoroethylene crystallized on slow cooling from the melt. Scale about $5 \mu \mathrm{m}$. (Ref. 30)

Figure 4. Lamellar edge of extended chain polytetrafluoroethylene. The rounding is believed to illustrate the steady state during crystallization. Scale about $2 \mu \mathrm{m}$. (Ref. 30)

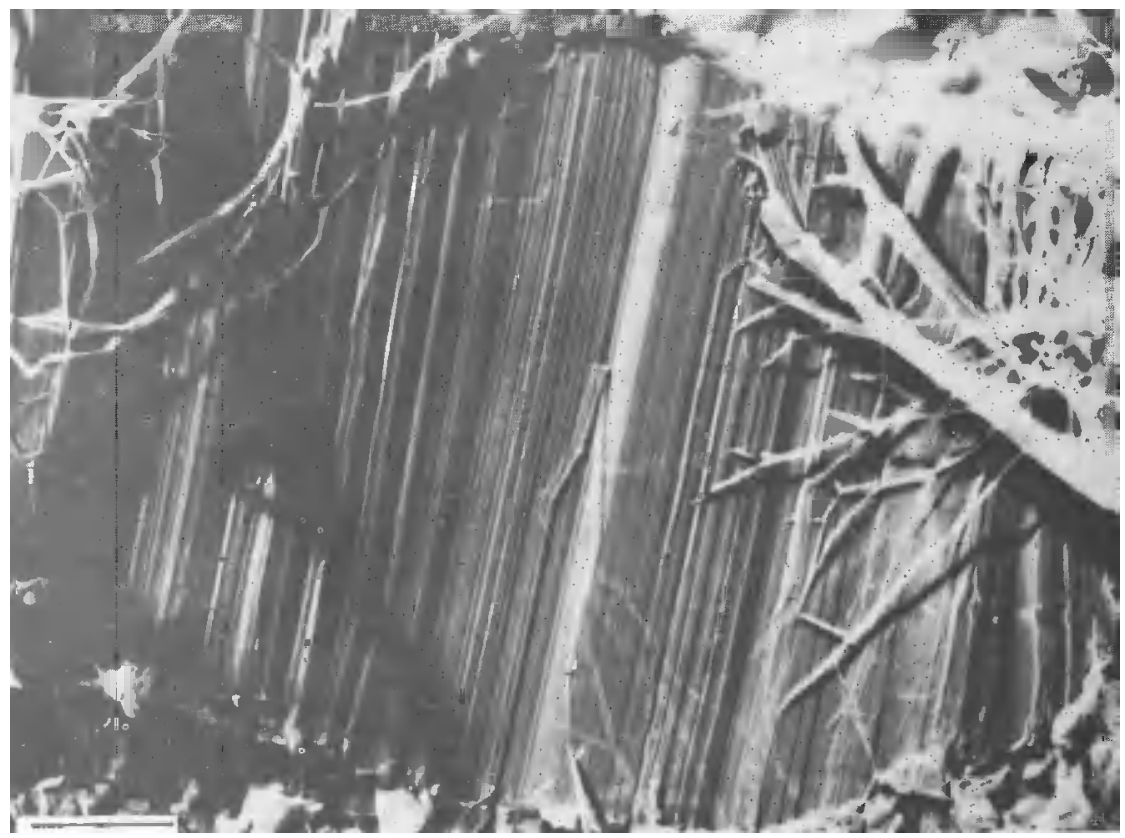

Figure 5. Electron micrograph of a fracture surface of polyethylene crystallized under $4800 \mathrm{~atm}$ pressure at $226^{\circ} \mathrm{C}$. Scale $0.5 \mu \mathrm{m}$. (Ref. 11) 
pressure and a parallel increase in crystallization temperature by about $60^{\circ} \mathrm{C}$ is necessary to accomplish chain extension.

Crystallization of polyethylene from solution under elevated pressure does not yield chain extension, even at similar crystallization temperatures and higher pressures ${ }^{18}$. Also, annealing of once chain-folded polyethylene crystals under conditions of pressure and temperature which on crystallization from the melt leads to chain extension, does not lead to similar chain extensions ${ }^{14,16}$. Seeding with extended chain crystals has also only little effect on the overall chain extension of a crystallizing melt or solution ${ }^{19}$. Finally, extended chain crystals with large side surface area, such as can be found in the rough surface portion on fracture, refold on annealing below the melting poing of the bulk crystal as is shown in Figure $6^{28}$. All these observations are in accord with a
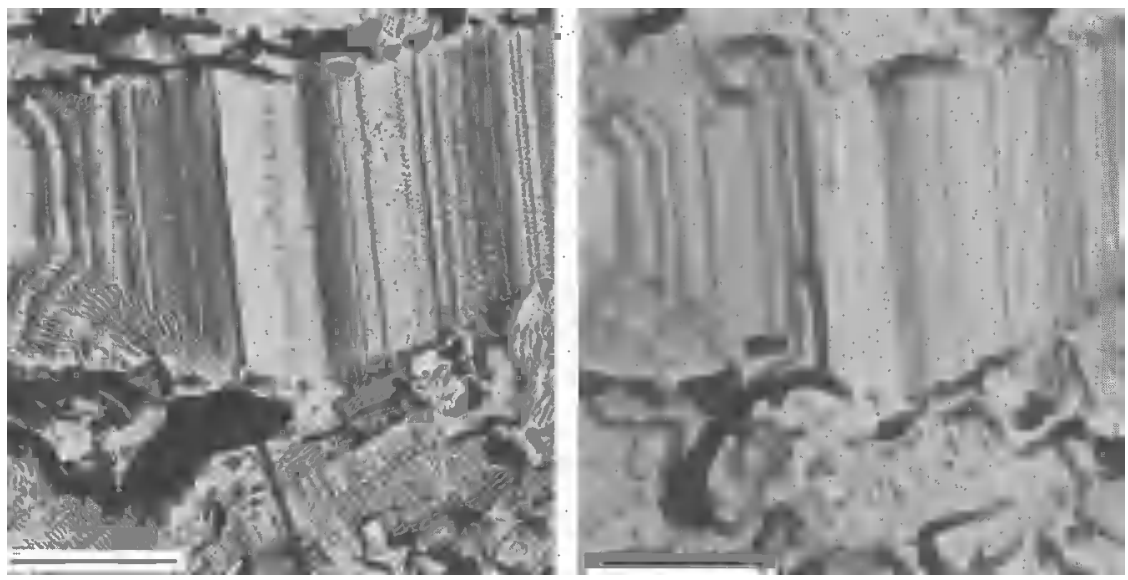

Figure 6. Electron micrograph of a replica of an extended chain surface of polyethylene before (right) and after (left) annealing at $120^{\circ} \mathrm{C}$ for three minutes. The newly developed "ripples" are folded chain lamellae grown epitactically. Chain direction of both ripples and extended chain lamellae are vertical in the figures. Scale $1 \mu \mathrm{m}$. (Ref. 28)

growth mechanism which has as its initial stage chain folded crystallization in a relatively defect form. On growth from solution or from the melt at atmospheric pressure, the defect initial crystals perfect themselves so fast that large scale chain extension cannot occur. On crystallization under pressure, however, the initial folded chain crystallization is slower ${ }^{20}$ and probably poorer so that the thickening in the defect solid state is sufficiently enhanced by the elevated temperature to result in extended chain crystals. On annealing of once-perfected folded chain lamellae, these conditions of thickening are not met. The starting material is already relatively well perfected, although with a small fold length. Annealing is only possible after disturbance of the initial crystal by a sufficient rise in temperature to create new defects by partial melting. The thickening is then in addition hindered by the limited availability of material laterally since all initial crystallization has already taken place. An initially folded lamella can gain in stability through solid state thickening only until its surface free energy 
reaches a minimum value. Transport of molecules across grain boundaries without loss of extension is unlikely as can be seen from experiments of refolding on the surface (see Figure 6).

The growth mechanism proposed is also in accord with the molecular weight segregation observed during extended chain crystallization ${ }^{14}$. During crystallization under pressure, rejection of short chain molecules was only accomplished up to molecular weights of about 10000 . Higher molecular weights were not segregated, and crystallized as relatively sharp-melting, mixed crystals. Crystallization at atmospheric pressure had quite similar fractionation behaviour ${ }^{21}$. Figure 7 illustrates a comparison of a broad molecular weight

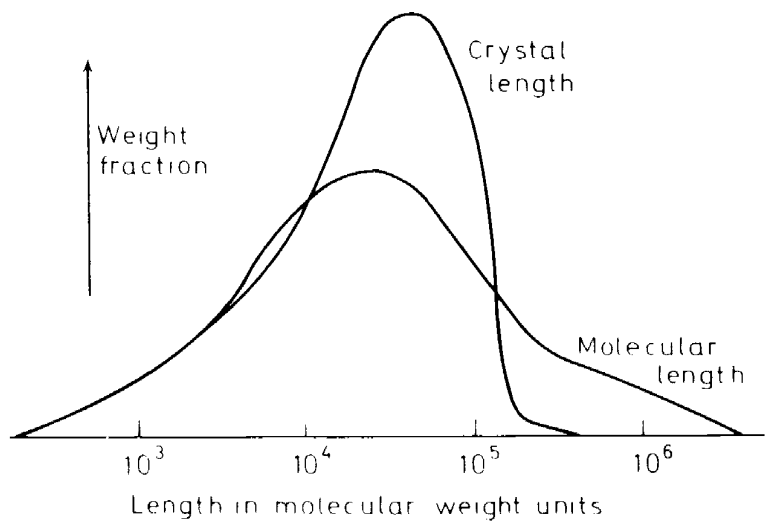

Figure 7. Molecular length distribution of a typical broad molecular weight polyethylene. Superimposed is the crystal length distribution after crystallization at elevated pressure, also in molecular weight units ( $1 \AA$ corresponds to 11 molecular weight units). (Ref. 14)

polyethylene sample crystallized in an extended chain form. For molecular lengths corresponding to molecular weights up to about 10000, lamellar thickness and molecular length agree, indicating fully extended chain crystals. Above 10000 molecular weight, the molecular length is not a measure of lamellar thickness: rather, various lengths occur in the same thinner mixed crystals. Melting experiments ${ }^{14}$ have supported the microscopic evidence of Figure 7.

Recently, extended chain crystals have been grown from melts of polychlorotrifluoroethylene ${ }^{22}$. Rather perfect extended chain crystals could be obtained by the application of only $1000 \mathrm{~atm}$ on crystallization from the melt.

Another extended chain material crystallizable from the polymeric melt is selenium. Its fracture surface is shown in Figure 8 and also indicates a chain folding process followed by chain extension ${ }^{23}$. In addition, however, selenium can also react chemically by chain opening and remaking. Considerable evidence has been collected that this added annealing process alters chain folds after initial folding and extension. Annealing by fold opening and remaking of tie molecules will strengthen the grain boundaries of the otherwise rather weak crystal aggregates. A similar annealing process was shown to be possible in poly(ethylene terephthalate) where chain folds can be removed in hydrolysis 


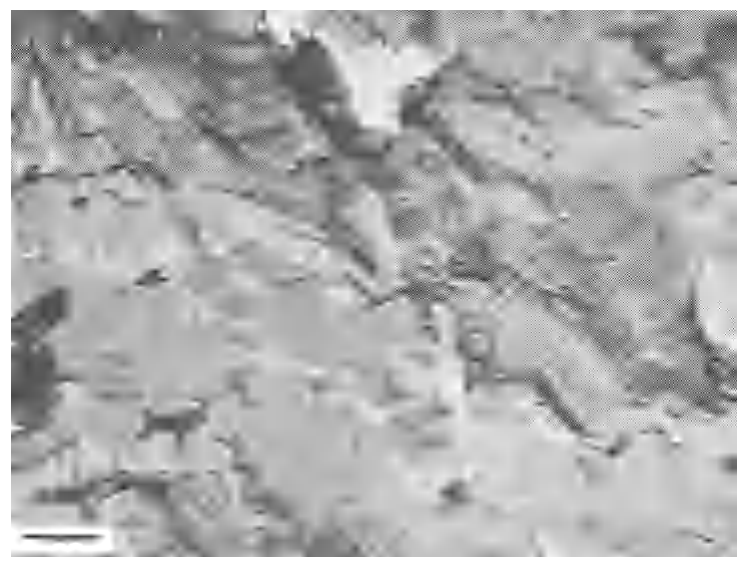

Figure 8 . Fracture surface of selenium crystallized on slow cooling from the melt. Scale $5 \mu \mathrm{m}$. (Ref. 30)

and tie molecules remade by repolymerization below the melting temperature of the initially folded chain crystals ${ }^{24}$.

In summary, chain extension of once chain-folded crystals is possible under appropriate conditions via a solid state thickening process. The best conditions for this thickening are present immediately after crystallization where added folded chain crystallization can supply the needed polymer and where perfection of the initial crystal has not yet occurred. A second process is possible when chain molecules can be reversibly broken. For selenium and poly(ethylene terephthalate) fold removal and production of tie molecules by chemical reaction was demonstrated. Since the initial crystallization of molten or dissolved macromolecules must be folded with a relatively small fold period, this route to extended chain crystals is somewhat limited.

\section{Extended chain crystal growth by crystallization during polymerization}

None of these limitations on chain extension exists when crystallization occurs during polymerization. Small monomer molecules can in this case be added directly to the growing polymer crystal. No a priori reason exists in this case for chain folding. Also, since the macromolecule never exists in the liquid state, there is no limitation on molecular weight caused perhaps by handling of high viscosity melts or solutions. Growth of the polymer crystal can under such conditions occur from monomer molecules in the gaseous, liquid (dissolved or molten), or even solid state.

Crystallization of macromolecules from solid monomers has, despite the obvious limitations, received most attention in the past. Many topochemical reactions were found so that polymerization in the solid state was able to provide new materials. Usually, however, the polymer produced was in the amorphous state or crystallized only after polymerization was at least partially complete $^{5}$. Recently some rather special monomers were crystallized and 
polymerized topotactically, Wegner ${ }^{25}$ succecúd in polymerizing several derivatives of 2,4-hexadiyn-1,6-diols in the solid state initiated by light or heat. Details were investigated for the crystal form I of poly (2,4-hexadiyn-1,6-diol bis-phenyl urethane). The monomer crystals which are colourless change rapidly to a deep red with a parallel increase in tensile strength (from 1 to $10000 \mathrm{~kg} \mathrm{~cm}^{-2}$ ). The crystal morphology remains, although some disorder is evident in the $\mathrm{x}$-ray diffraction pattern.

Similarly Hasegawa, Y. Suzuki, F. Suzuki and Nakanishi ${ }^{26}$ could polymerize crystalline 2,5-distyrylpyrazine and related compounds by the action of sunlight or ultraviolet light. Single crystals of the monomer have been converted to highly crystalline polymer samples with related morphology. Once molten, the polymer is difficult to recrystallize.

Two examples of crystallization during polymerization from the liquid phase are the formation of polyphosphates on dehydration of primary phosphates and the formation of polyoxymethylene from formaldehyde in aqueous or alcoholic solution. The main problem of crystallization in both cases is the nucleation step $^{27}$. Before simultaneous crystallization and polymerization can begin, a nucleus of molecules of sufficient chain length must grow from oligomers. The oligomers for this nucleation are grown in solution in a normal step reaction. Experimental evidence indicates ${ }^{27}$ that nucleation occurs as soon as approximately the decamer appears in reasonable yield ( 1 to 2 mole \%). This concentration is reached after about $50 \%$ of the reaction is completed. At this point the reaction mechanism changes to a direct chain reaction. Remaining monomer, dimer, etc. are added cooperatively to the crystal or nucleus surface. Instead of a further slow increase in molecular weight, crystals of high molecular weight are formed next to a melt of low molecular weight. Except for the nucleation step this reaction is similar to crystal growth of low molecular weight compounds. Similarly, one does expect isometric crystals from such reactions. Figure 9 illustrates some crystals of lithium polyphosphate grown in this fashion. The fracture surface of these crystals is shown in Figure 10. It is similar to the

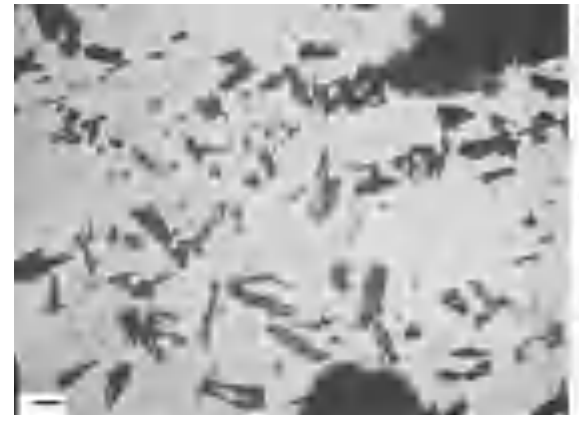

FIG. 9

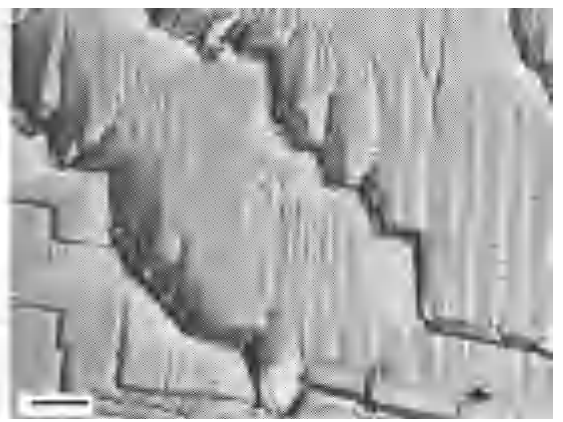

FIG. 10

Figure 9. Single crystals of $\mathrm{LiPO}_{3}$ grown on heating $\mathrm{LiH}_{2} \mathrm{PO}_{4}$. Scale about $1 \mu \mathrm{m}$. (Mininni and Wunderlich. Unpublished. 1969)

Figure 10. Electron micrograph of a replica of a fracture surface of a $\mathrm{LiPO}_{3}$ crystal. Scale about $2 \mu \mathrm{m}$. (Mininni and Wunderlich. Unpublished. 1969) 
fracture surface of the extended chain lamellae discussed above. The striations are in all cases a result of the anisotropy of the motifs of the crystal ${ }^{28}$.

Reactions from the gas phase can naturally also be found to yield under proper conditions extended chain crystals. A particularly well analysed case is the epitactic growth of nylon 6 crystals on its own monomer ${ }^{29}$. This epitaxy was accomplished by polymerization of the reactive gaseous monomer at $168^{\circ} \mathrm{C}$ in the presence of monomer crystals which contain unreactive zwitterions. The resultant polymer chains are oriented $\pm 55^{\circ}$ to the crystallographic $b$-axis of the monomer. The polymer crystal was nucleated in register with the monomer orientation on the (001) surface. Once nucleated, the polymer keeps the or ientation despite considerable mismatch of lattice parameters of monomer and polymer crystals. In addition, the nucleation step seems to be limited to the initial growth period so that lateral growth is restricted. The epitaxial polymer appears in the form of extended chain needles. Figure 2 shows needles or fibres of polytetrafluoroethylene grown from the gas phase ${ }^{30}$. In these fibres the chain orientation is also along the fibre axis and little growth seems to occur in the lateral direction with time.

Fibrous growth seems to be particularly common ${ }^{27}$ in polymers which grow inherently with chain reaction mechanisms. Many such reactions have been reviewed recently ${ }^{5}$. Two cases had to be distinguished: simultaneous polymerization and crystallization, and successive polymerization and crystallization. All cases discussed up to now in this section are believed to be of the first category where the locations of polymerization and crystallization are identical or in the immediate vicinity. Successive crystallization and polymerization allows increasing separation between polymerization and crystallization sites until finally no interaction occurs between the two sites. Even further removed, it is possible that the chains take on a random conformation so that crystallization has again to occur via chain folding. All stages of separation may occur in the case of polymerization of $p$-xylylene from the gas phase at increasingly lower temperatures, a reaction at present under investigation in our laboratory ${ }^{31}$.

In summary, growth of extended chain crystals by crystallization during polymerization is possible. No a priori restrictions to crystal length seem to exist for such growth. Restrictions to the reaction process may, however, lead to fibrillar morphology. Overall, only a beginning has been made to investigate the problems involved with simultaneous and successive polymerization and crystallization.

\section{DESCRIPTION OF EXTENDED CHAIN CRYSTALS}

Extended chain crystals have the same crystal structure as folded chain crystals. From the modes of formation described above, it has been deduced that extended chain crystals may have a lamellar, isometric, or fibrous habit. Only the isometric habit is close to the equilibrium dimensions. In all lamellae the chain direction is close to normal to the lamellar surface so that, because of the large chain extension, surface effects are also rather small. The fibrous habit is the least stable crystal form. 


\section{Lamellar habit}

The lamellar habit of extended chain polyethylene is the only one analysed in more detail ${ }^{15}$. It is similar to the folded chain habit ${ }^{32}$ out of which it evolved by chain extension. Figures 11 and 12 show the appearance of a typical extendedchain polyethylene crystal sample under a polarizing microscope and an

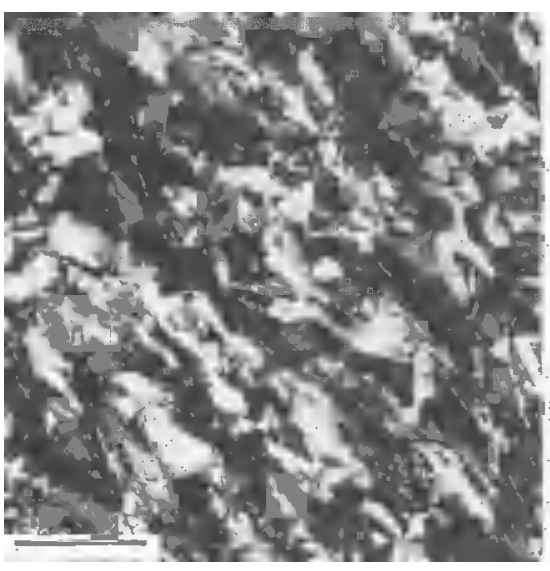

FIG. 11

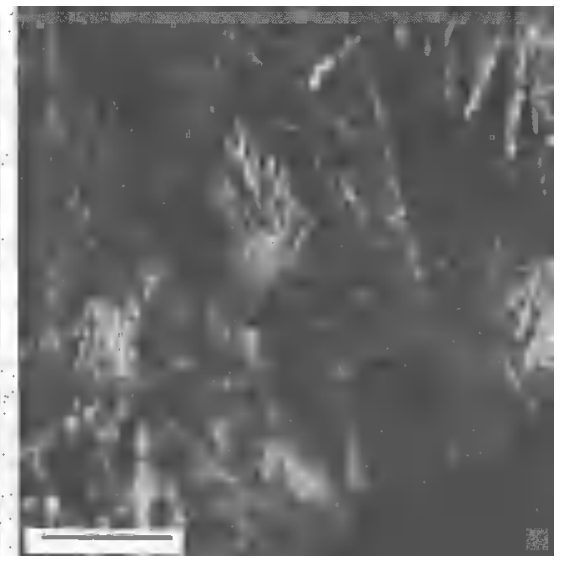

FIG. 12

Figure 11. Microtome-cut section of an extended chain crystal preparation of polyethylene between crossed polarizers. Scale $100 \mu \mathrm{m}$. (Ref. 15)

Figure 12. Microtome-cut section of an extended chain crystal preparation of polyethylene observed under an interference microscope. Scale $25 \mu \mathrm{m}$. (Ref. 15)

interference microscope after cutting with a microtome to $2 \mu \mathrm{m}$ thickness. Single lamellae can be seen edgewise. Estimation of the birefringence establishes the orientation of the chains at right angles to the lamellar surface. There seems to be no limit imposed on the lateral dimensions other than the availability of space and crystallizable material.

A common feature of all extended chain crystals is the striated appearance of their fracture surface (see Figures 3-5, 10). The striations run in all investigated samples parallel to the molecular chain axes. The striation direction has been established by birefringence measurement and by electron diffraction on occasional material included in the replica ${ }^{11}$. The elevation of the striations over an average surface height was shown to be random with a step height of only 1 to 3 chain thicknesses ${ }^{28}$. Fibrous and isometric extended chain crystals show similar fracture surfaces (see Figure 10). Even highly drawn amorphous polymers fracture similarly. Further evidence that the striations are surface effects only comes from the annealing of the surface to smoothness, a process which starts as much as $40^{\circ}$ below the melting point ${ }^{28}$. On refracturing, a new striated surface was created.

The lamellar surface is usually close to the crystallographic (001) surface and relatively featureless when observed under the electron microscope. The distribution of lamellar sizes shown for example in Figure 7 is much broader 


\section{EXTENDED CHAIN CRYSTALS OF POLYMERS}

than found in solution-grown folded chain lamellae which are usually within limits of \pm 10 to $20 \AA$. The packing of the lamellae can occur in three modes: parallel, at right angles, and at a small angle. Parallel packing is the natural way of stacking lamellae because of geometry. The right angle packing can be linked to epitaxial nucleation of new lamellae on the otherwise crystallographically inactive (001) surface which would orient the chain axis into the lamellar surface. Finally, small angle orientation is connected with the spherulitic growth habit of most crystalline polymer samples. Branching can occur easily on the growth face by introduction of folds in the body of the lamellae. Because of the inability of the crystal to accommodate folds, a branch will occur with the two daughter lamellae splaying apart at a small angle. A spherulitic extended chain sample of polyethylene and the branching frequency are illustrated in Figures 13 and 14.

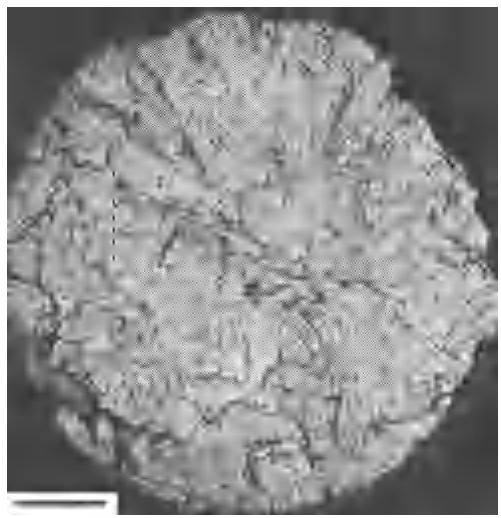

Figure 13. Electron micrograph of the fracture surface of pressure crystallized polyethylene as in Figures 11 and 12 showing the spherulitic development. Scale $10 \mu \mathrm{m}$. (Ref. 15)

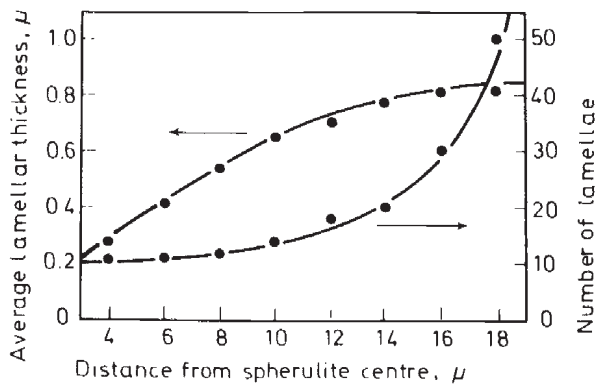

Figure 14. Graph of the change of lamellar thickness and number of lamellae of a $53^{\circ}$ sector of the spherulite in Figure 13 as a function of distance from the spherulite centre. (Ref. 15) 


\section{BERNHARD WUNDERLICH}

The deformation behaviour by slip parallel to $1001 \mid$ is seen in Figures 15 and 16. The surface of the crystals is in these two figures, and also the following two figures, decorated with "ripples", folded chain lamellae grown epitaxially to illustrate the chain direction of the underlying extended chain crystals. Another frequently observed defect are kink-bands as shown in Figure $17(a)$ and $(b)$. It is likely that these kink-bands in polyethylene are introduced during crystal growth ${ }^{28}$. while in polytetraflurorethylene they were linked to deformation ${ }^{33}$.

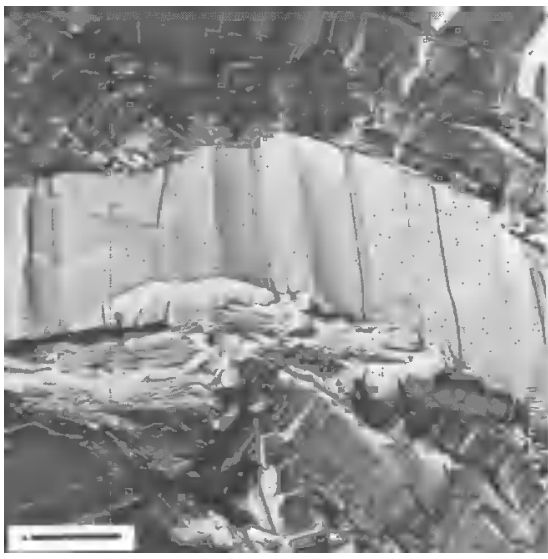

FIG. 15

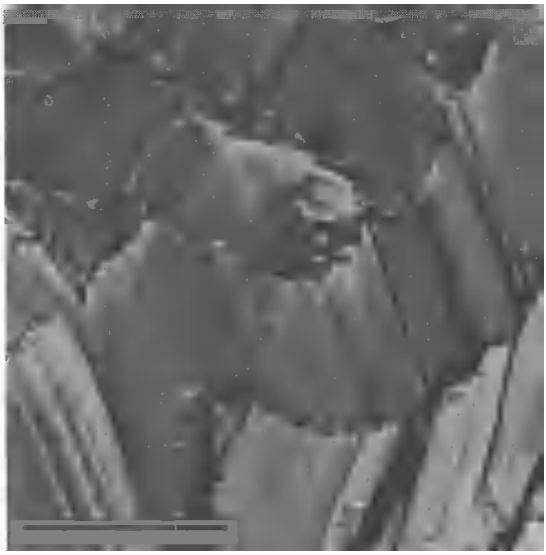

FIG. 16

Figure 15. Fracture surface of polyethylene showing a bent lamella due to slip along $|001|$. The surface is ripple decorated. Scale $1 \mu \mathrm{m}$. (Ref. 15)

Figure 16. Fracture surface of polyethylene showing strong deformation at points of lamellar intersection. As in Figure 15 the deformation is caused by slip along $1001 \mid$. Scale $1 \mu \mathrm{m}$. (Ref. 15)

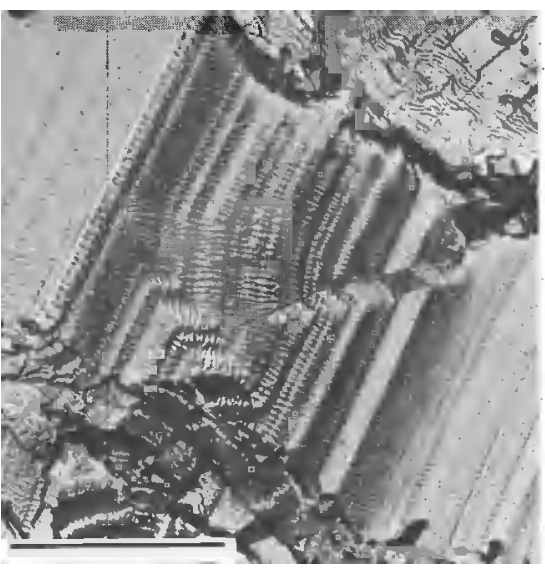

a

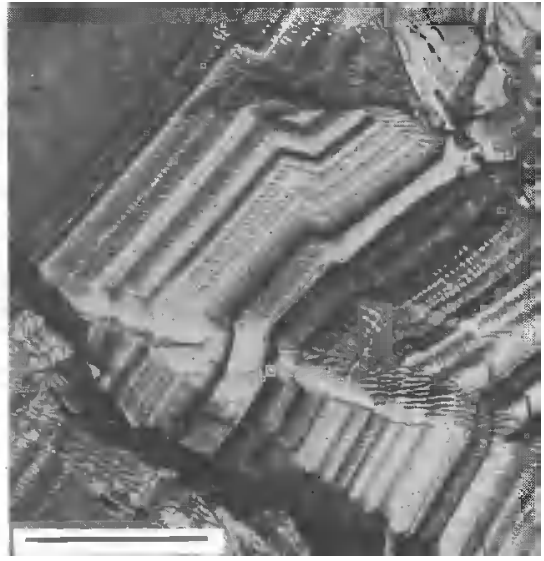

b

Figure $17(a)$ and $(b)$. Fracture surfaces of lamellae showing kink bands decorated with ripples to establish the molecular chain direction. Scale $1 \mu \mathrm{m}$. (Ref. 15) 


\section{Isometric and fibrous habit}

Too little is known about these two habits for a detailed analysis. On fracture both will show the typical striations described above. Of particular interest are, in both cases, the molecular weight distributions since they can, in conjunction with the crystal dimensions, give information on internal defects. For isometric polyphosphates the crystal defect and size dependence of molecular weights has been discussed ${ }^{34}$.

The fibrous habit can take on any degree of perfection and may also contain considerable amounts of chain folding; it is derived from mechanically deformed folded chain lamellae. When the fibrils have a lower melting point than the corresponding folded chain lamellae, they refold on annealing as shown in Figure 6 and in several other Figures as "ripple" decoration where the fibres created on a fracture surface are recrystallized.

Branching of the fibrous habit leads also to spherulitic growth. The resulting spherulites are, however, of opposite molecular chain orientation to the lamellar spherulites. In fibrous extended chain crystals radial chain orientation is observed, while in the lamellar spherulites tangential chain orientation occurs. Examples of radial chain orientation are observed for selenium, polyoxymethylene, and some proteins.

Epitaxial fibrous growth as described above for polycaprolactam ${ }^{29}$ has also a $90^{\circ}$ different chain orientation from the usual epitaxy of folded chain polymers as is illustrated for example by the ripples.

\section{PROPERTIES OF EXTENDED CHAIN CRYSTALS}

Extended chain crystals with close to equilibrium morphology should also show equilibrium properties. Melting points ${ }^{13,}{ }^{14}$, phase relationships ${ }^{14}$, heat capacities $^{35}$, and dissolution temperatures ${ }^{36}$ were found to be close to the theoretically expected values. The melting point when measured sufficiently slowly is as sharp as is exhibited by well-crystallized organic small molecules. The heat capacity corresponds up to the melting point to the values computed from the vibrational spectrum of the polymer. In the case of broad molecular weight distributions the lower molecular weight fraction acts as a solvent for the higher and reduces the melting point as can be predicted from the phase diagram of polymers and diluents. Other structure-insensitive properties like heat of fusion, modulus, density, $\mathrm{x}$-ray diffraction, and IR absorption were also shown to be close to the expected values for equilibrium crystals.

The larger size and well-formed features on slowly crystallized lamellae of polyethylene resulted in a minimum of tie molecules for not too high molecular weight. The crystals were thus well separated and the sample took on a brittle character. Light tapping with a hammer would powder the samples by fracture. As soon as the molecular weight was increased and/or the crystallization conditions changed to increase tie molecules, the samples changed to the usual tough character.

Heating extended-chain crystals at faster rates leads to superheating ${ }^{37-40}$. Figure 18 illustrates the superheating of polyoxymethylene crystallized from trioxane vapour. Only at the slowest heating rate does the melting peak show a sharply melting compound. From the known thermal conductivity one can 


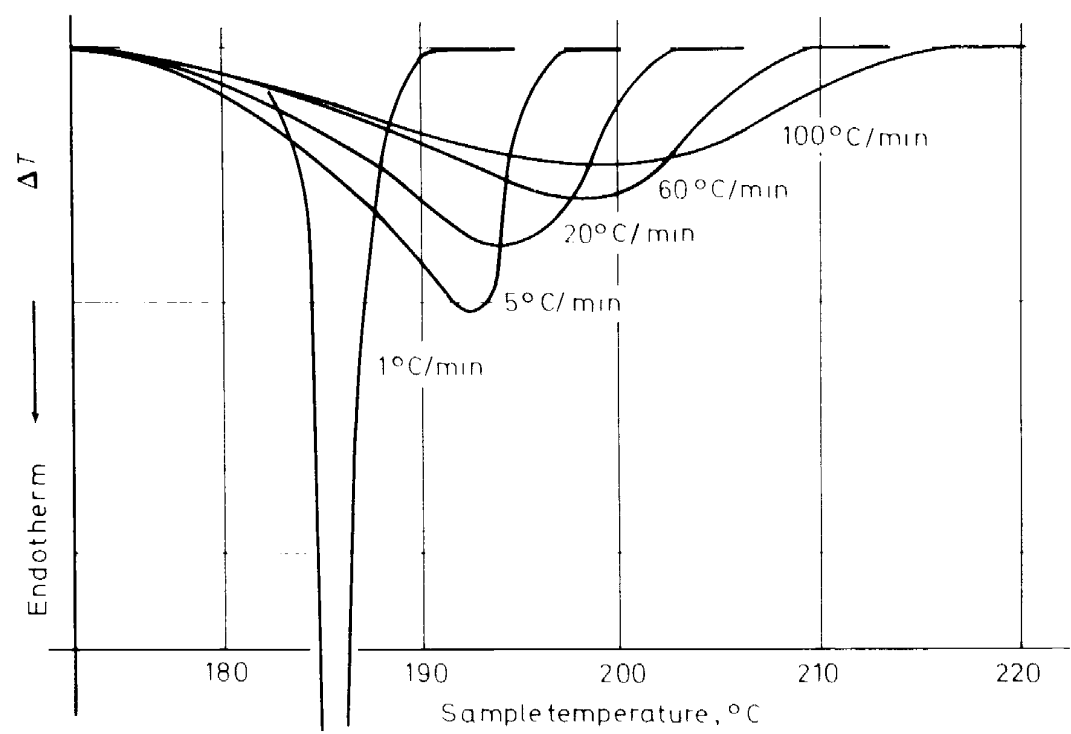

Figure 18. Differential thermal analysis curve of the melting of extended chain crystals of polyoxymethylene at different heating rates. (Ref. 40)

deduce that thermal equilibrium is still established throughout all these experiments. The interior of the crystals may thus reach a temperature up to $30^{\circ}$ above the melting point. The reason for superheating may be the necessary sequential melting of chain atoms. In addition, it was likely that entropy restrictions can temporarily increase the melting point ${ }^{41}$. Recently it has also been shown that remaining chain folds possibly cause a slowing down of the melting. On etching the lamellar surfaces with nitric acid the superheating character could be partially reduced ${ }^{42}$.

\section{ACKNOWLEDGEMENTS}

Much of the work reported from our Laboratory was supported by the National Aeronautics and Space Administration, the Office of Naval Research, and the National Science Foundation. Present support is given by the Allied Chemical Corporation.

\section{REFERENCES}

P. J. Flory. Statistical Mechanics of Chain Molecules, Interscience, New York (1969).

2 G. Wulff. Z. Krist. 34, 449 (1901).

3 B. Wunderlich and T. Davidson. J. Polymer Sci. A2, 7, 2043 (1969).

${ }^{4}$ L. Mandelkern. Crystallization of Polymers, McGraw-Hill, New York (1964).

5 B. Wunderlich. Fortschr. Hochpolymer. Forsch. 5, 568 (1968).

${ }^{6}$ H. Staudinger and R. Signer. Z. Krist. 70, 193 (1929).

7 K. H. Storks. J. Am. Chem. Soc. 60, 1753 (1938).

${ }^{B}$ P. H. Geil. Polymer Single Crystals, Interscience, New York (1963).

${ }^{9}$ C. W. Bunn. A. J. Cobbold and R. P. Palmer. J. Polymer Sci. 28, 365 (1958).

10 B. Wunderlich and T. Arakawa. J. Polymer Sci. A. 2. 3697 (1964). 


\section{EXTENDED CHAIN CRYSTALS OF POLYMERS}

1 P. H. Geil, F. R. Anderson, B. Wunderlich and T. Arakawa. J. Polymer Sci. A, 2, 3707 (1964).

12 T. Arakawa and B. Wunderlich. J. Polymer Sci. A2, 4, 53 (1966).

13 T. Arakawa and B. Wunderlich. J. Polymer Sci. C, 16, 653 (1967).

14 B. Wunderlich and T. Davidson. J. Polymer Sci. A2, 7, 2043 (1969).

15 B. Wunderlich and L. Melillo. Makromol. Chem. 118, 250 (1968).

16 E. W. Fischer and H. Puderbach. Kolloid-Z. Z. Polymere 235, 1260 (1969).

17 D. V. Rees and D. C. Bassett. J. Polymer Sci. A2, 9, 385 (1971).

18 B. Wunderlich. J. Polymer Sci. A 1, 1245 (1963).

19 B. Wunderlich and C, M. Cormier. J. Phys. Chem. 70, 1844 (1966).

20 T. Davidson and B. Wunderlich. J. Polymer Sci. A2, 7, 377 (1969).

21 F. R. Anderson. J. Appl. Phis. 35. 64 (1964).

${ }^{22}$ Y. Miyamoto, C. Nakafuko and T. Takemura. To be published.

${ }^{23} \mathrm{M}$. Coughlin and B. Wunderlich. To be published.

24 A. Miyagi and B. Wunderlich. To be published.

${ }^{25}$ G. Wegner. Z. Naturforsch. 24b, 824 (1969).

${ }^{26}$ M. Hasegawa, Y. Suzuki, F. Suzuki and H. Nakanishi. J. Polymer Sci. A 1, 7, 743, 753 (1969).

27 B. Wunderlich. Angew. Chem. 80, 1009 (1968); Int. Ed. Engl. 7, 912 (1968).

28 B. Wunderlich, L. Melillo, C. M. Cormier, T. Davidson and G. Snyder. J. Macromol. Sci. B 1. 485 (1967).

${ }^{29}$ E. M. Macchi, N. Morosoff and H. Morawetz. J. Polymer Sci. A1, 6, 2033 (1968).

${ }^{30}$ L. Melillo and B. Wunderlich. To be published.

${ }^{31} \mathrm{~S}$. Kubo and B. Wunderlich. To be published.

32 A. Keller. Rept. Progr. Phys. 31, 623 (1968).

${ }^{33}$ C. J. Speerschneider and C. H. Li. J. Appl. Phys. 34, 3004 (1963).

34 aJ. R. van Wazer and C. F. Callis in F. A. G. Stone and W. A. G. Graham (Eds.). Inorganic Polymers, Academic Press. New York (1962).

${ }^{b}$ H. Malmgreen and O. Lamm. Z. Anorg. Allgem. Chem. 252, 256 (1944).

${ }^{35}$ B. Wunderlich. J. Phys. Chem. 69, 2078 (1965).

${ }^{36}$ C. M. Cormier and B. Wunderlich. J. Polymer Sci. A2, 4, 666 (1966).

${ }^{37}$ E. Hellmuth and B. Wunderlich. J. Appl. Phys. 36, 3039 (1965).

${ }^{38}$ E. Hellmuth, B. Wunderlich and J. M. Rankin. J. Appl. Polymer Sci. 2, 101 (1966).

${ }^{39}$ M. Jaffe and B. Wunderlich in R. F. Schwenker and P. D. Garn (Eds). Thermal Analysis, Vol. 1, p. 387. Academic Press, New York (1969).

${ }^{40}$ M. Jaffe and B. Wunderlich. Kolloid Z.Z. Polymere 216-217, 203 (1967).

${ }^{41}$ H. G. Zachman. Kolloid-Z. Z. Polymere 206, 25 (1965).

42 K.-H. Illers. Makromol. Chem. 118, 88 (1968). 